\title{
Valoración de la rugosidad superficial de una resina fluida sometida a diferentes $\mathrm{pH}$ salivales
}

\author{
Assessment of the surface roughness of a flowable resin \\ subjected to different salivary $\mathrm{pH}$
}

\author{
Marjorie Gissela Amaya-Sánchez (C) ${ }^{1 a}$, Alejandra-Cabrera (b) ${ }^{1 b}$, Ana Del Carmen Armas-Vega (b) 1c
}

\section{RESUMEN}

Objetivo: Determinar la rugosidad superficial y estabilidad de color de una resina nanohíbrida sometida a diferentes $\mathrm{pH}$ salivales en diferentes tiempos. Materiales y métodos: estudio de tipo experimental, comparativo, in vitro, en el cual se utilizaron 45 discos de resina nanohíbrida EA2 (OPALLIS FGM) de $10 \mathrm{~mm}$ de diámetro y $1 \mathrm{~mm}$ de espesor. Fueron distribuidos aleatoriamente en tres grupos $(n=15)$, cada uno de los discos fue sometido a evaluación de su rugosidad mediante un rugosímetro digital (TESTER SRT-6200) y color, mediante colorímetro (Tooth Color Comparator). Los discos de acuerdo a su grupo fueron sumergidos en $18 \mathrm{ml}$ de saliva artificial modificada con ácido cítrico hasta obtener saliva de $\mathrm{pH} 5,6$ y 7 respectivamente y colocados en reservorios específicos mantenidos a $37^{0} \mathrm{C}$ de temperatura, según especificaciones de la norma ANSI/ADA n ${ }^{0} 66$. Transcurridos 168 y 504 horas de almacenamiento, fueron realizadas una segunda y tercera evaluación. Para el analisis estadisitico se utilizaron las pruebas de Friedman y Kruskal Wallis Resultados: Los tres $\mathrm{pH}$ salivales modificaron la rugosidad superficial del material usado, de forma más evidente el $\mathrm{pH} 5$, observándose un incremento de la rugosidad superficial con el tiempo de exposición a las sustancias con los diferentes $\mathrm{pH}$ analizados $(\mathrm{p}<0,01)$, el color mostró un evidente incremento de 4 tonos en todas las muestras. Conclusiones: El incremento de rugosidad fue proporcional con el tiempo e inversamente proporcional con el pH de forma más evidente con pH5. El mecanismo de evaluación del color no permitió obtener un dato conclusivo.

Palabras claves: Saliva artificial; $\mathrm{pH}$, Resinas compuestas; Colorimetría (Fuente: DeCS BIREME)

\begin{abstract}
Objective: To determine the surface roughness and color stability of a nanohybrid resin subjected to different salivary $\mathrm{pH}$ at different times. Materials and methods: experimental, comparative, in vitro study, in which 45 discs of EA2 nanohybrid resin (OPALLIS FGM) of $10 \mathrm{~mm}$ in diameter and $1 \mathrm{~mm}$ in thickness. They were randomly distributed in three groups $(n=15)$, each of the resin discs was subjected to an evaluation of its roughness by means of a digital roughness meter (TESTER SRT-6200) and color by colorimeter (Tooth Color Comparator). The discs according to their group were immersed in $18 \mathrm{ml}$ of artificial saliva modified with citric acid until obtaining saliva of $\mathrm{pH} 5,6$ and 7 respectively and placed in specific reservoirs maintained at $37^{\circ} \mathrm{C}$, according to the specifications of the ANSI / ADA n0 standard. 66, after 168 and 504 hours of storage, a second and third evaluation was carried out. For the statistical analysis, the Friedman and Kruskal Wallis tests were used. Results: The three salivary pHs modified the surface roughness of the material used in a more evident way, $\mathrm{pH} 5$, observing an increase in surface roughness with the time of exposure to substances with different $\mathrm{pH}$ analyzed $(\mathrm{p}<0.01)$, the color showed an evident increase of 4 tones in all the samples. Conclusions: The increase in roughness was proportional with time and inversely proportional with the $\mathrm{pH}$, more evident with $\mathrm{pH}$. The color evaluation mechanism did not allow obtaining a conclusive data.
\end{abstract}

Keywords: Artificial saliva; pH; Composite resin; Colorimetry (Source: MeSH NLM)

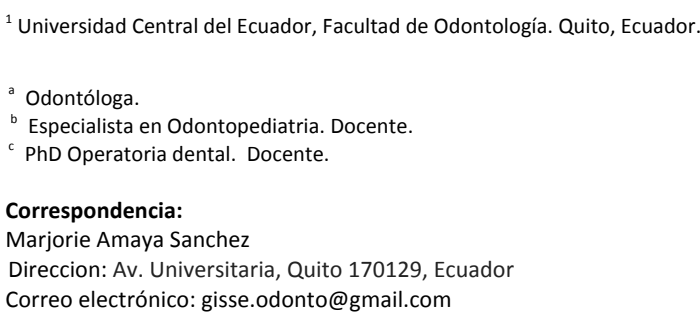

Este es un artículo de acceso abierto distribuido bajo la licencia Creative Commons Atribución 4.0 Internacional (CC BY 4.0) https://creativecommons.org/licenses/by/4.0/deed.es

Citar como: Amaya Sanchez MG, Cabrera A, Armas Vega A. Valoracion de la rugosidad superficial de una resina fluida sometida a diferentes pH salivales. KIRU. 2021;18(2):75-81. https://doi.org/10.24265/kiru.2021.v18n1. 


\section{INTRODUCCIÓN}

La era de las resinas modernas inicia en 1962 con el desarrollo de un nuevo monómero ${ }^{(1)}$, llamado Bisfenol-A-Glicidil Metacrilato (Bis-GMA) y un agente de acoplamiento o silano entre la matriz de resina y las partículas de relleno ${ }^{(2)}$, a partir de ese momento las resinas compuestas han sido testigos de numerosos avances, llegando en los últimos años a una nueva tecnología, resultando resinas compuestas microhíbridas y nanohíbridos ${ }^{(3)}$, donde la combinación de nanofillers y nanoclusters reduce los espacios entre las partículas de relleno al tiempo que proporciona la máxima carga de relleno, lo que resulta en una mayor longevidad del pulido de la superficie ${ }^{(4)}$.

Las resinas aún están lejos de ser consideradas ideales debido a sus limitaciones, incluidas deficiencias mecánicas y elevada susceptibilidad a la degradación en el ambiente oral ${ }^{(5)}$, que se constituye un mecanismo complejo cuya velocidad depende de varios factores, entre ellos la matriz polimérica, partículas de relleno y otros procesos, tales como la absorción de agua en el interior, ciclos térmicos y mecánicos, con la subsecuente formación y propagación de grietas ${ }^{(6,7)}$. Las condiciones ambientales orales críticas relacionadas con cambios de $\mathrm{pH}$ y humedad, pueden aumentar la biodegradación de la resina compuesta en el tiempo ${ }^{(8)}$, observándose que el agua es absorbida por el polímero, mismo que actúa como un agente plastificador, altera parcialmente la energía de cohesión de las cadenas poliméricas ${ }^{(9,10)}$. Así, la presencia de agua desencadena la disminución prematura de las propiedades mecánicas del polímero ${ }^{(11)}$, que con el paso del tiempo resultará en la hidrólisis de sus moléculas, condenando así su estructura ${ }^{(12)}$.

La rugosidad de la superficie causada por el desgaste y la degradación química también puede afectar el brillo y en consecuencia, aumentar la tinción extrínseca del material en la retención de placa y microorganismos, desencadenantes directos de caries secundarias y fallas en la restauración ${ }^{(13)}$. A esto se suma el hecho que las superficies rugosas influirían en la dispersión de la luz y el grado de reflexión, afectando el tono o translucidez de la superficie del material ${ }^{(14)}$. Observándose que un $\mathrm{pH}$ más bajo produce un efecto adverso en la resistencia al desgaste de los compuestos de resina ${ }^{(8)}$. La saliva por la presencia del bicarbonato constituye el elemento responsable del proceso buffer, que intenta mantener el $\mathrm{pH}$ entre $6,7^{\text {y }} 7,5^{(15,16)}$, este mecanismo en determinadas circunstancias se encuentra alterado, principalmente por la ingesta desproporcionada de alimentos o bebidas con $\mathrm{pH}$ en extremo ácido o básico, higiene bucal deficiente, poco control de placa bacteriana, enfermedad periodontal entre otros ${ }^{(6,17)}$.

Cuando el $\mathrm{pH}$ de la saliva disminuye y se mantiene en el tiempo es un factor predisponente para la degradación de la matriz polimérica ${ }^{(8)}$, donde el tiempo constituye un determinante importante para tener en cuenta, diferentes estudios demostraron que frente a caries o enfermedad periodontal, el $\mathrm{pH}$ de la saliva se convierte en ácido (18), constituyéndose así la saliva en uno de los factores determinantes para el éxito de las restauraciones directas de resina, ocasionando su deterioro el excesivo consumo de comidas ácidas o bebidas gaseosas y alcohólicas ${ }^{(19)}$. Frente a lo expuesto resulta básico conocer el grado de repercusión que tienen los $\mathrm{pH}$ salivales sobre una resina nanohíbrida (OPALLIS, FGM), sobre su rugosidad y color.

\section{MATERIALES Y MÉTODOS}

Se plantea una investigación de tipo experimental, comparativo e in vitro, en una muestra constituida por 45 discos ${ }^{(20)}$ de resina nanohíbrida EA2 (OPALLIS FGM), confeccionadas con la ayuda de una matriz a base de poli acrílico de $10 \mathrm{~mm}$ de diámetro y $1 \mathrm{~mm}$ de espesor colocadas sobre una loseta de vidrio, empleando una espátula de titanio y una lámpara de luz LED Curing Light/ Ultradent polimerizando durante 20 segundos según las indicaciones del fabricante, a una longitud de onda de $420 \mathrm{~nm}-480 \mathrm{~nm}$, a una distancia de $2 \mathrm{~mm}$ entre la muestra y la lámpara dental, regulada y calibrada la intensidad de la luz para mantenerse constante con un radiómetro.

Confeccionados los cuerpos de prueba, estos fueron sometidos a pulido utilizando discos flexibles de óxido de aluminio (Sof-Lex; 3M ESPE) de forma secuencial, grano grueso, mediano, fino y ultra fino, para eliminar la capa rica en resina y suavizar la superficie de la muestra. Empleando cada disco a un pulido de 20 segundos con movimiento circular de manera interrumpida para evitar el sobrecalentamiento y los cambios de superficie consecuentes, utilizando una pieza de mano de baja velocidad de 12,000 r.p.m. Una vez confeccionados y pulidos los discos de resina, se dividieron aleatoriamente en tres grupos $(n=15)$ y fueron colocados en envases de vidrio ámbar, para evitar la exposición a la luz y al medio externo.

Se evaluó su rugosidad mediante rugosímetro digital (TESTER SRT-6200). Para este propósito, cada muestra se fijó cuidadosamente con plastilina 
sobre un soporte metálico. Se colocó con mucho cuidado la punta de diamante del palpador del rugosímetro en la parte más recta de la muestra de resina y se realizó un recorrido a una velocidad de palpación $0.135 \mathrm{~mm} / \mathrm{s}$ con una longitud de onda límite $0,25 \mathrm{~mm}$, realizándose en cada disco de resina 7 medidas, las muestras permanecían a $37^{\circ}$ C realizando mediciones a las 168 y 504 horas, registrando los resultados en cuadros previamente elaborados.

La evaluación en cuanto al color de cada disco, fue ejecutado por el investigador principal mediante un el colorímetro digital (Tooth Color Comparator) previa calibración, mismo que registra el color dental basándose en guía de colores VITAPAN classical A1-D4 Shades (VITA16), en el cual se asignó con puntajes del 1 al 16 para cada tonalidad, en donde el puntaje 1 corresponde al tono B1 que es el más claro y el puntaje 16 corresponde al tono $\mathrm{C} 4$ que es el más oscuro.

Los discos se almacenaron en cajas Petri, debidamente identificadas, dentro de las cuales se colocó $60 \mathrm{ml}$ de saliva artificial (Salivsol) en cajas Petri, la saliva fue modificada en cuanto a su pH a través de la incorporación de ácido cítrico hasta obtener un $\mathrm{pH}$ de 5,6 y 7 siguiendo la metodología previamente aplicada $^{(20,4,21,8)}$, comprobando estos valores mediante un $\mathrm{pH}$ metro digital, conservando las sustancias en envases a una temperatura de $37{ }^{\circ} \mathrm{C}$, según las especificaciones propuestas por la norma ANSI/ADA $n^{0} 66^{(22,4)}$, al transcurrir las 168h considerada como tiempo 1 de almacenaje fue realizada una segunda evaluación en cuanto a su rugosidad y color ${ }^{(4,21,8)}$ así como una tercera al completar las 504 horas (7). Los resultados obtenidos fueron registrados en tablas específicamente diseñadas y sometidas a un análisis estadístico mediante el programa SPSS mediante un software libre R v3.5.2 a través del teste de Kruskal Wallis y Friedman.

\section{RESULTADOS}

Con respecto a la rugosidad superficial el análisis descriptivo determinó diferencias numéricas entre los datos, donde el tiempo 3 , los $\mathrm{pH} 5$ y 6 generarían los valores de rugosidad promedio más altos, observando una variabilidad de la rugosidad mayor a las $504 \mathrm{~h}$ en comparación con las $168 \mathrm{~h}$ y la inicial, lo que dificulta realizar pruebas de hipótesis tipo ANOVA, pudiendo visualizarse mediante el diagrama de caja y bigote variabilidad de los datos en cada $\mathrm{pH}$ que a priori se incrementa en el tiempo (Gráfico 1).

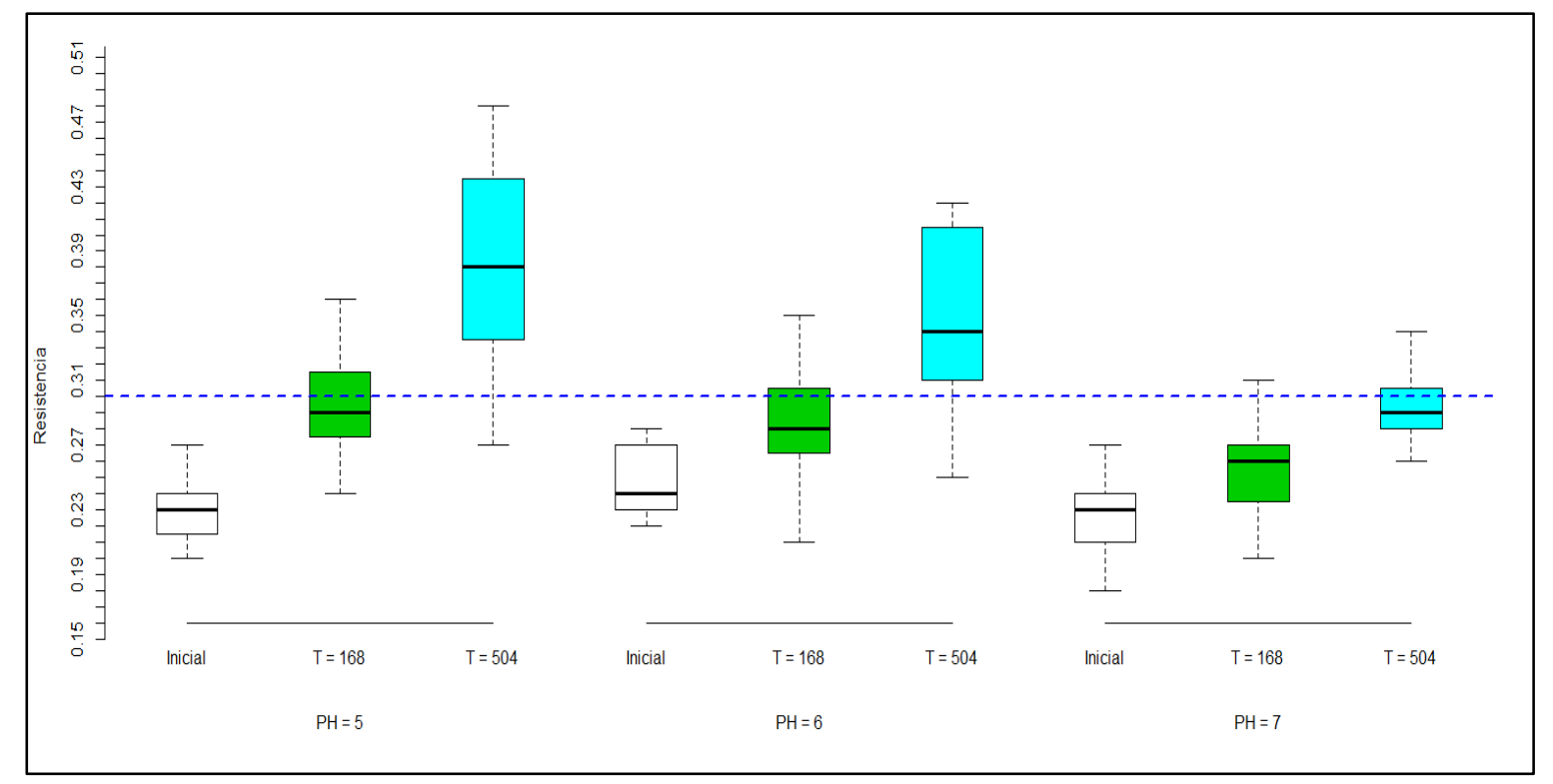

Figura 1. Distribución de las rugosidades según pH y tiempo 
Con la finalidad de evaluar las diferencias significativas, se construyen los intervalos de confianza del $95 \%$ para la rugosidad media, los cuales no solo confirman la tendencia ascendente de la rugosidad en el tiempo para cada $\mathrm{pH}$; sino que además muestran que en los tres $\mathrm{pH}$ la rugosidad crece significativamente a medida que el tiempo transcurre (Gráfico 2).

El pH 7 si cambia la rugosidad en el tiempo, pero el crecimiento es más lento comparando con los $\mathrm{pH} 6$ y $\mathrm{pH} 5$ que crecen de manera más rápida, llegan a valores más altos en el mismo tiempo.

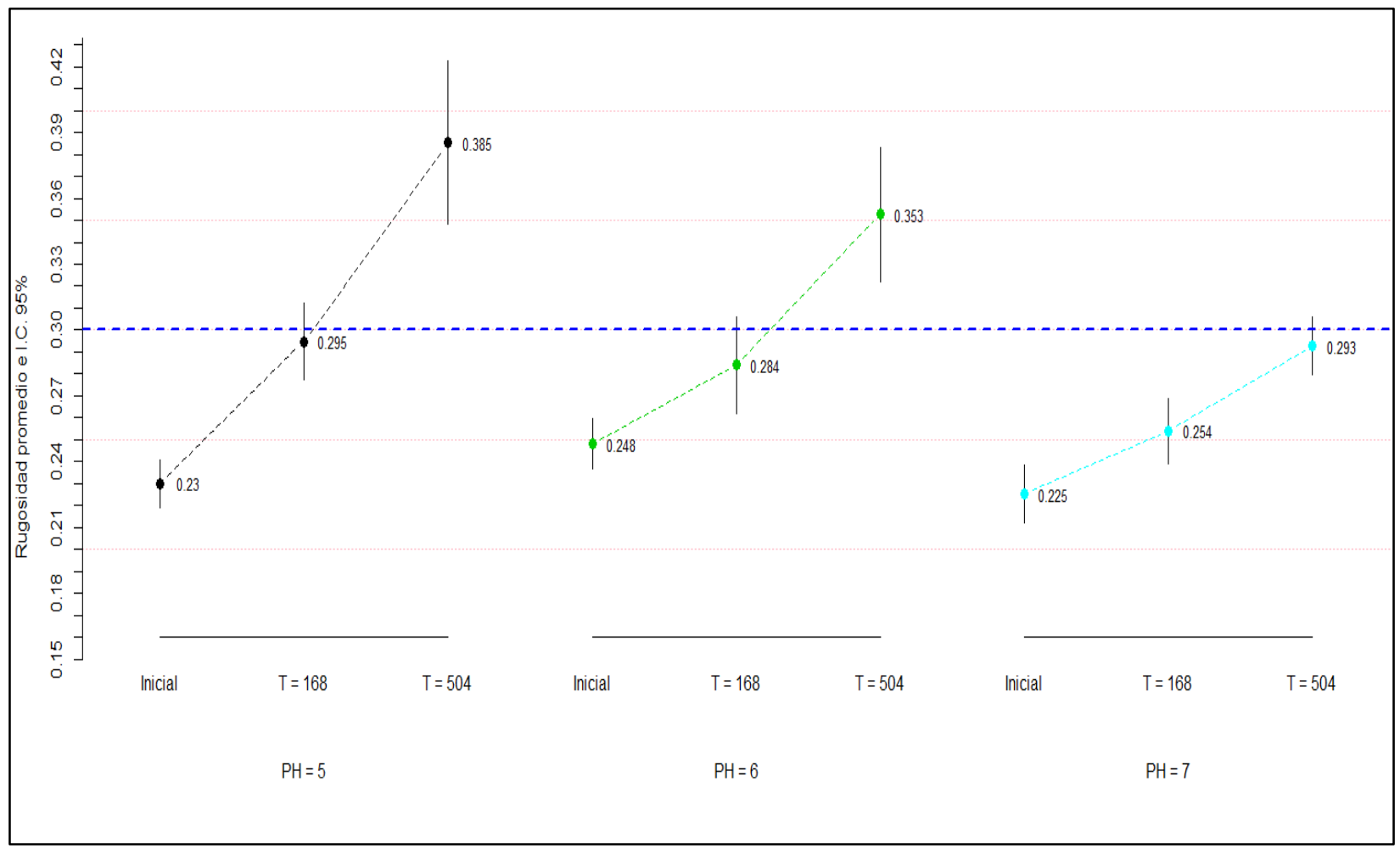

Figura 2. Rugosidades promedio e intervalos de $95 \%$ según pH y tiempo

Para cada uno de los valores de $\mathrm{pH}$ fue realizado una prueba de Friedman para evaluar muestras relacionadas, observándose que los valores $p$ en los tres $\mathrm{pH}$ resultan ser 0.000 , indicando que, en efecto para cada $\mathrm{pH}$, existe diferencia significativa entre las rugosidades al contrastarse entre tiempos. Para evaluar la existencia o no de diferencias significativas entre las rugosidades en similares tiempos, pero contrastados por $\mathrm{pH}$, se realiza la prueba de Kruskal-Wallis, encontrando que las rugosidades iniciales guardan diferencia significativa ( $p$-valor $=0.036)$, es decir, las distribuciones de los datos, en particular las rugosidades iniciales no fueron similares. De la gráfica de intervalos de confianza se detecta que la rugosidad promedio de $\mathrm{PH}=6$ fue mayor que las rugosidades iniciales de $\mathrm{pH} 5 \mathrm{y} \mathrm{pH} 7$, así al relacionar con el tiempo de almacenamiento se observa las rugosidades mantienen diferencia significativa entre los tres niveles de $\mathrm{pH}$ ( $\mathrm{p}$-valor $=$ 0.004) que se mantiene cuando el tiempo se incrementa $(p=0.000)$,es decir, las tres rugosidades promedio son diferentes siendo que con $\mathrm{pH} 5$ el incremento de rugosidad en el tiempo es más veloz que con los otros valores de $\mathrm{pH}$, de hecho, el incremento de rugosidad con $\mathrm{pH} 7$ es más lento y no llega a valores relativamente altos respecto de los otros niveles de $\mathrm{pH}$. Con respecto al color el valor inicial para todos los especímenes fue $A 2$, que según la luminosidad tiene un puntaje de 5, mientras que el valor final varió de acuerdo a cada espécimen y se le asignó el puntaje correspondiente, observando que con un $\mathrm{pH} 5$ se presentó variación de los tonos de las muestras comparando las $168 \mathrm{~h}$ de contacto con el pH y el tiempo inicial, así mismo de 504 horas con el 
tiempo inicial, observándose en los tres $\mathrm{pH}$ una variación en la iluminación que dificulta un análisis mediante ninguna prueba estadística inferencial ni obtención de medias por la homogeneidad de valores obtenidos.

\section{DISCUSIÓN}

Los resultados encontrados en el estudio demostraron que a mayor tiempo de exposición de los discos de resina en saliva artificial con $\mathrm{pH} 5$ produjo mayores cambios en su rugosidad y variación de color, observación ya reportada en estudios previos ${ }^{(9,4,21)}$, asociado a la composición química de los materiales $y$ una posible degradación de su matriz ${ }^{(6,9,23)}$, resultado de reacciones complejas entre diferentes factores ${ }^{(6)}$, como el consumo de alimentos ácidos, bebidas alcohólicas, bebidas suaves y energéticas ${ }^{(24)}$, con la influencia de adsorción o absorción de colorantes de fuentes exógenas en el color de estos materiales ${ }^{(9,4,21)}$, resultado de reacciones complejas entre diferentes factores ${ }^{(6)}$, como el consumo de alimentos ácidos, bebidas alcohólicas, bebidas suaves y energéticas ${ }^{(24)}$, con la influencia de adsorción o absorción de colorantes de fuentes exógenas en el color de estos materiales ${ }^{(9,4,21)}$. De esta manera el tipo de matriz de la resina, el porcentaje y la distribución del tamaño de partícula de los rellenos incorporados, tipo de fotoiniciador y el porcentaje de dobles enlaces restantes y polimerización incompleta $(10,25,14)$ se muestran como elementos determinantes de un mayor cambio o no a causa de sustancias acidas, observando ser el tiempo un elemento de gran influencia sobre la rugosidad ${ }^{(19)}$.

En estudios previos un almacenamiento de discos de resina compuesta en contacto con bebidas con diferentes $\mathrm{pH}$, demostró como periodos de 7 días de contacto con sustancias de $\mathrm{pH}$ de 2,47 produjeron lesión en la integridad del material evaluado ${ }^{(4)}$, con una relación inversamente proporcional donde a menor $\mathrm{pH}$ mayor rugosidad ${ }^{(8)}$ lo que incrementa la adsorcion y con ello cambios en el color y superficie del material, que clínicamente puede fácilmente ser detectada por el paciente $(26,27,13)$. Con respecto al color, las muestras mostraron alteraciones de color en todos los grupos, incluso cuando sumergidos en sustancias con $\mathrm{pH} 7^{(15,16)}$ decoloración asociada al envejecimiento del material ${ }^{(9,20)}$, a la composición del material donde el componente inorgánico y tamaño de partícula muestra una influencia determinante $^{(3,6,9)}$, no permitiendo a los polímeros formar oligómeros y finalmente monómeros ${ }^{(7)}$, con mayor repercusión en tiempos prolongados ${ }^{(21)}$.
Una de las limitaciones más importantes del estudio, fue su naturaleza, in vitro, donde las condiciones bucales no pueden ser reproducidas de manera fiel y por tanto los resultados cuentan con un elemento de sesgo más que evidente, a esto se suma la dificultad de contar en nuestro estudio con una forma de evaluación del color precisa, que permita captar los cambios detectados clínicamente, pese al grosor de cada espécimen e iluminación del ambiente, por lo que nuevos estudios requieren ser realizados considerando estos elementos y tratando de simular las condiciones orales en que estos materiales se desarrollan.

Pese a las limitantes reportadas, es básico como clínicos conocer las implicaciones existentes de las resinas compuestas y su desempeño a nivel bucal, para poder decidir sobre el material a ser usado y para asesorar al paciente en cuanto a los cuidados a ser tomados tras la colocación de este tipo de materiales en boca. Es bien sabido que el éxito de cualquier procedimiento dental no depende de un solo factor; sin embargo, limitar los elementos negativos que pueden influenciar sobre estos resultados es importante.

\section{CONCLUSIONES}

Los tres grupos de resina nanohíbrida expuestos a diferentes $\mathrm{pH}$ salivales aumentaron su rugosidad superficial, siendo más evidente estos cambios cuando el pH disminuye, el mecanismo de evaluación de cambios de color en los discos de resina no permitió obtener un resultado conclusivo.

\section{BIBLIOGRAFÍA}

1. Suarez R, Lozano F. Comparación de la dureza superficial de resinas de nanotecnología, según el momento del pulido: in vitro. Rev Estomatol Herediana [Internet]. 2014; 24(1): p. 11-16.

2. Chan $\mathrm{KH}$, Mai $\mathrm{Y}$, Kim H, Tong KC, Ng D, Hsiao JC. Review: Resin Composite Filling. PMC. [Internet]. 2010; 3(2): p. 1228-1243.

3. Demirci M, Tuncer S, Sancakli H, Tekçe N, Baydemir C. Five-year Clinical Evaluation of a Nanofilled and a Nanohybrid Composite in Class IV Cavities. Operative Dentistry [Internet]. 2018; 43(3): p. 261271.

4. Tavangar M, Bagheri R., Kwon TY, Mese $A$, Manton DJ. Influence of beverages and surface roughness on the color change of resin composites. Journal of Investigative 
and Clinical Dentistry [Internet]. 2018; 1(1): p. 1-8.

5. Satomi T, Pereira T, Vieira F, Ofelia j, Fernando F, Goulart Goyotá F. Restauraciones posteriores con resina compuesta: relato de caso clínico. KIRU [Internet]. 2014; 11(2): p. 175-179.

6. Arana BE, Achury JL, Coral N, Pulgarin LM, Sepúlveda W. Evaluación del color y rugosidad superficial de resina compuesta después de someterse a enjuagues bucales. Scielo [Internet]. 2014; 5(13): p. 15.

7. Bhargava A, Sharma D, Majumdar D, Bhargava $A$, Bansal $M$, Meel $P$. Original article "effects of temperature of beverages on ardness, surface roughness and color stabilty of resin composite -an in vitro study. IOSR Journal of Dental and Medical Sciences (IOSR-JDMS) [Internet]. 2016; 8(5): p. 18-23.

8. Ilday $\mathrm{N}$, Bayindir $\mathrm{YZ}$, Erdem V. Effect of three different acidic beverages on surface characteristics of composite resin restorative materials. Materials Research Innovations [Internet]. 2010; 14(5): p. 385391.

9. Hwang S, Chung SH, Lee JT, Kim YT, Kim $Y J$, Oh S, Yeo IS. Influence of Acid, Ethanol, and Anthocyanin Pigment on the Optical and Mechanical Properties of a Nanohybrid Dental Composite Resin. Materials (Basel) [Internet]. [citado Jul 2018];11(7): 1234. 2018; 11(7): p. 1234.

10. Ulian G, Lia R, Charantola M, Batista E, Kiyoshi S, Wang L. Impact of filler size and distribution on roughness and wear of composite resin after simulated toothbrushing. Scielo [Internet]. [citado Oct 2012];20(5): 1 - 7. 2012; 20(5): p. 1-7.

11. Coreño-Alonso J, Méndez-Bautista $M$. Relationship between structure and properties of polymers. Scielo [Internet]. 2010; 4(21): p. 1-11.

12. Herrera DR, Kose-Jr C, Villa-Verde F, Stanislawczuk R, Reis A, Loguercio AD. Clorhexidina como alternativa para maximizar la longevidad de restauraciones adhesivas. Rev Estomatol Herediana [Internet]. 2010; 20(2): p. 78-84.

13. Itanto $B$, Usman $M$, Margono $A$. Comparison of surface roughness of nanofilled and nanohybrid composite resins after polishing with a multi-step technique. IOP Publishing [Internet]. 2017; 884: p. 1-7.

14. Lepri C, Palma R. Surface roughness and color change of a composite: Influence of beverages and brushing. Dental Materials Journal [Internet]. 2012; 31(4): p. 689-696.

15. Calatrava L. La saliva: una ventana para el diagnóstico. RevVenezlnvestOdont IADR [Internet]. 2014; 2(2): p. 65-74.

16. Aguirre A, Narro SF. Perfil salival y su relación con el índice CEOD en niños de 5 años. Revista Odontológica Mexicana [Internet]. 2016; 20(3): p. 159-165.

17. Sánchez JC, Urzúa I, Faleiros S, Lira J, Rodríguez G, Cabello R. Capacidad buffer de la saliva en presencia de bebidas energéticas comercializadas en Chile, estudio in vitro. Rev Clin Periodoncia Implantol Rehabil Oral [Internet]. 2015; 8(1): p. 24-30.

18. Barrios C, Vila V, Martinez S, Encina A. Ph Salival como factor asociado a la caries dental. Revista Facultad De Odontología [Internet]. 2017; 10(1): p. 13-19.

19. Rodríguez D, Bonilla P, Aillón E, Tello G. Efecto de barnices fluorados sobre el esmalte erosionado a través de microscopia de fuerza atómica: Estudio in vitro. Revista Odontología [Internet]. 2017; 19(1): p. 55-74.

20. Cengiz $S$, Yüzbasioglu $E$, Inanç Cengiz $M$, Velioglu N, Sevimli G. Color Stability and Surface Roughness of a Laboratory Processed Composite Resin as a Function of Mouthrinse. Journal of Esthetic and Restorative Dentistry [Internet]. 2015; 27(5): p. 314-321.

21. Moon JD, Son SA, Jung $\mathrm{KH}$, Kwon $\mathrm{YH}$, Park JK. Effect of immersion into solutions at various $\mathrm{pH}$ on the color stability of composite resins with different shades. PubMed [Internet]. 2015; 40(4): p. 270276.

22. ANSI/ADA. Procedimientos en Microbiología Clínica. [Online]; 2009. Disponible en: https://www.seimc.org/contenidos/documen toscientificos/procedimientosmicrobiologia/ seimc-procedimientomicrobiologia32.pdf.

23. Khan A, Siddiqui A. Effect of different $\mathrm{pH}$ solvents on micro-hardness and surface topography of dental nano-composite: An in vitro analysis. Pakistan Journal of Medical Sciences [Internet] 2015; 31(4): p. 854-859. Available: https://scihub.se/10.12669/pjms.314.7517

24. Dasilva M, Vitti R, Sinhoreti M, Consani R, Dasilva-Júnior And Tonholo J. Effect of alcoholic beverages on surface roughness and microhardness of dental composites. Dental Materials Journal [Internet]. 2016; 35(4): p. 621-626. 
25. Lamas C, Alvarado S, Angulo G.
Importancia del acabado y pulido en

27. Lavín S, Toro UG. Cuantificación de la Importancia del acabado y pulido en
restauraciones directas de resina compuesta en piezas dentarias anteriores. Reporte de Caso. Scielo [Internet]. 2015; 25(2): p. 145-151.

26. Bashetty K, Joshi S. The effect of one-step and multi-step polishing systems on surface texture of two different resin composites. PubMed [Internet]. 2010; 9(6): p. 1-7.

\author{
Marjorie Amaya Sanchez (1) http://orcid.org/0000-0001-5574-9762 \\ gisse.odonto@gmail.com \\ Marina Alejandra Cabrera Arias \\ alecabreta_arias@hotmail.com \\ D http://orcid.org/0000-0002-8403-2995 \\ Ana Armas Vega \\ (1) http://orcid.org/0000-0003-3800-8166 \\ ana_del_ec@yahoo.es
}

Copyright $\odot$ La revista. La revista Kiru es publicada por la Facultad de Odontología de la Universidad de San Martín de Porres, en Lima, Perú. 